\title{
Significado de calidad de vida en pacientes con terapia de hemodiálisis: un estudio fenomenológico
}

\author{
Leslie Herbias Herbias, Romina Aguirre Soto, Hernán Bravo Figueroa, Lissette Avilés Reinoso
}

Facultad de Enfermería. Universidad Andrés Bello. Santiago de Chile. Chile

\section{Resumen}

La calidad de vida es uno de los aspectos importantes a abordar en pacientes en hemodiálisis. Objetivo: Comprender el significado de calidad de vida para personas que son sometidas a tratamiento de sustitución renal con hemodiálisis. Metodología: Estudio cualitativo fenomenológico descriptivo. Se realizaron entrevistas en profundidad a 12 personas en hemodiálisis. Las entrevistas fueron grabadas y transcritas textualmente. El análisis se realizó de acuerdo a Streubert, se resguardaron criterios de rigor y se contó con la aprobación del Comité científico de la Facultad de Enfermería de la Universidad Andrés Bello. Resultados: Calidad de vida fue descrita a través de tres categorías comprensivas: Salud, Familia y Actividades de la vida diaria, que involucra ámbitos físicos, psicológicos y sociales. Los participantes significan la calidad de vida cómo Seguir viviendo, ya que producto de la terapia pueden continuar con su vida. Conclusiones: Comprender lo que significa calidad de vida permite ampliar la mirada, fortalecer el vínculo con la familia y considerar lo crucial de esta terapia en la vida de las personas en hemodiálisis. Se sugiere fortalecer la relación enfermera-paciente con el fin de otorgar cuidados seguros y de calidad a los usuarios y sus familias.

\begin{tabular}{|c|}
\hline Correspondencia: \\
Lissette Avilés Reinoso \\
Diplomada en Hemodiálisis, Magíster en Enfermería \\
Profesor Asistente, Facultad de Enfermería, \\
Universidad Andres Bello, Chile \\
Sazié $2212,6^{\circ}$ piso. Santiago, Chile. \\
Dirección Postal: 8370136 \\
E-mail: lissette.aviles@unab.cl
\end{tabular}

PALABRAS CLAVE

- HEMODIÁLISIS

- CALIDAD DE VIDA

- ENFERMERÍA

- ESTUdIO CUALITATIVO

Meaning of quality of life in patients on hemodialysis therapy: a phenomenological study

\begin{abstract}
Quality of life is one of the most important aspects in the management of patients in hemodialysis. Aim: To understand the meaning of quality of life in people who require hemodialysis. Methodology: Qualitative research with phenomenological approach. Thorough interviews were done to 12 patients in hemodialysis. Interviews were recorded and transcripted, and these were performed according to Streubert, rigor criteria were protected, and the Ethics Commitment of Faculty of Nursing, Universidad Andres Bello approved it. Results: Quality of life was described in three comprehensive categories: Health, Family and Daily Activities. These outcomes comprised physical, psychological and social aspects. For patients the term "quality of life" means Keep living, because thanks to the treatment they can continue with their lives. Conclusion: To understand the meaning of Quality of life allows us wide the view of the phenomenon, and to strengthen the role of the relatives in the treatment. These essential elements need to be considered in order to support patients in hemodialysis. Thus, it is necessary to build a stronger bond between the nurse and the patient with the target to improve nursing interventions in patients and relatives.
\end{abstract}




\section{KEYWORDS}

- HEMODIALYSIS

- QUALITY OF LIFE

- NURSING

- QUALITATIVE RESEARCH

\section{Introducción}

Calidad de vida es un concepto complejo y multidimensional del cual aún no hay consenso, y que a su vez es de vital importancia en pacientes con Enfermedad Renal Crónica Terminal (ERCT) en tratamiento con hemodiálisis. En Estados Unidos, cerca de 26 millones de adultos sufren de ERCT y otros millones están en riesgo de padecerla. Esto, debido al envejecimiento progresivo de la población y el aumento de otras enfermedades crónicas, como por la Diabetes Mellitus ${ }^{1}$. En España, se cifran en 4 millones el número de personas que padecen enfermedad renal crónica de las cuales aproximadamente 51.000 de ellas requiere tratamiento sustitutivo renal ${ }^{2}$. En Chile, 18.160 personas reciben tratamiento con hemodiálisis, de los cuales el $41 \%$ reside en la Región Metropolitana ${ }^{3}$.

Calidad de vida, es definida de acuerdo a la Organización Mundial de la Salud (OMS) como "la percepción que un individuo tiene de su lugar en la existencia, en el contexto de la cultura y del sistema de valores en los que vive y en relación con sus expectativas, sus normas e inquietudes ${ }^{\prime \prime 4}$. Se trata por lo tanto, de un concepto amplio que está influido por la salud física del sujeto, su estado psicológico, su nivel de independencia y sus relaciones sociales, entre otros. Sin embargo, cada persona puede tener una concepción diferente al respecto, lo que se refleja en la variedad de opiniones y de percepciones entre los diferentes grupos de edad, sexo, cultura y obviamente, estado de salud.

En un estudio desarrollado en Brasil ${ }^{5}$, los sujetos que se dializaban entre 1 a 5 años presentaban una menor calidad de vida en comparación de los que llevaban menor tiempo sometidos a la terapia. Respecto del género, los hombres mostraban una mejor percepción de su calidad de vida, en particular, en la función emocional y en los efectos de la enfermedad en la vida cotidiana. Las dimensiones más afectadas eran la salud general y el peso de la enfermedad renal. Por otro lado, Seguí y Ramos $^{6}$ indican que la calidad de vida en pacientes con ERCT, es peor que en la población de referencia en todas las dimensiones. Además, concluyeron que existe una calidad de vida disminuida en los pacientes en tratamiento de diálisis siendo esta calidad peor en los mayores de 65 años y portadores de catéter temporal.

Enfermería, como pilar fundamental en el tratamiento de pacientes sometidos a terapia de sustitución renal, resulta de suma relevancia comprender lo que las personas viven, sienten y significan en relación a su calidad de vida. El cuidado se dirige hacia la satisfacción de las necesidades de los seres humanos desde una perspectiva holística y comprensiva, que incluye sus valores, creencias y percepciones. Conociendo estos aspectos se podría intervenir de forma más integral en el cuidado del paciente y su familia, de acuerdo a sus vivencias y necesidades.

El propósito de este estudio fue comprender el significado de calidad de vida de las personas que son sometidas a tratamiento de sustitución renal con hemodiálisis. La pregunta que guió este estudio fue ¿Cuál es el significado de calidad de vida en personas en tratamiento sustitutivo de hemodiálisis?

\section{Material y método}

Estudio cualitativo fenomenológico descriptivo, con esta metodología se busca comprender los fenómenos humanos a través de la descripción de la experiencia vivida, configurando unidades de significado y categorías comprensivas.

Los criterios de inclusión fueron: personas mayores de 18 años, en terapia de sustitución renal de hemodiálisis, asistentes a un centro de diálisis de la comuna de Ñuñoa de Santiago de Chile, y que hayan firmado el consentimiento informado. Este estudio contó con la aprobación del Comité Ético Científico de la Facultad de Enfermería de la Universidad Andres Bello.

La recolección de información se realizó en el mes de Octubre del año 2015 mediante entrevistas en profundidad, las que fueron grabadas y transcritas textualmente para su posterior análisis. El análisis se realizó de acuerdo a lo descrito por Streubert ${ }^{7}$. Primero se realizó una descripción personal del fenómeno de interés por parte del equipo de investigación, luego se notificaron los supuestos del investigador a través de escritos. Se comenzaron las entrevistas; se leyeron atentamente las transcripciones de las entrevistas para obtener un sentido general de la experiencia, luego se revisaron las transcripciones para descubrir el contenido y aprehender las relaciones esenciales. Se realizó la descripción del fenómeno y se devolvieron los resultados a los par- 
Tabla 1. descripción de los participantes.

\begin{tabular}{|c|c|c|c|c|c|c|c|c|}
\hline \multicolumn{9}{|c|}{ Ficha Participantes } \\
\hline & Sexo & Edad & Estado Civil & Hijos & $\begin{array}{c}\text { Nivel } \\
\text { educacional }\end{array}$ & Previsión & $\begin{array}{c}\text { Tiempo que lleva } \\
\text { dializándose } \\
\text { (<1 año, entre } 1 \text { y } 5 \\
\text { años, }>5 \text { años) }\end{array}$ & Comuna \\
\hline 1 & Masculino & 32 & Casado & 1 & $\begin{array}{l}\text { Básica } \\
\text { completa }\end{array}$ & FONASA B & Más 5 años & Peñalolén \\
\hline 2 & Masculino & 54 & Casado & 2 & $\begin{array}{c}\text { Media } \\
\text { completa }\end{array}$ & FONASA A & Entre 1 y 5 años & Macul \\
\hline 3 & Femenino & 55 & Casada & 4 & $\begin{array}{l}\text { Básica } \\
\text { completa }\end{array}$ & FONASA A & Entre 1 y 5 años & Nuños \\
\hline 4 & Masculino & 51 & Soltero & No & $\begin{array}{c}\text { Media } \\
\text { completa }\end{array}$ & FONASA B & Entre 1 y 5 años & Peñalolén \\
\hline 5 & Femenino & 74 & Viuda & 8 & $\begin{array}{c}\text { Básica } \\
\text { completa }\end{array}$ & FONASA B & Entre 1 y 5 años & Mcul \\
\hline 6 & Femenino & 56 & Soltera & 1 & $\begin{array}{c}\text { Básica } \\
\text { completa }\end{array}$ & FONASA B & Más 5 años & Conchali \\
\hline 7 & Masculino & 53 & Divorciado & 2 & $\begin{array}{l}\text { Superior } \\
\text { completa }\end{array}$ & FONASA B & Entre 1 y 5 años & Cerrillos \\
\hline 8 & Masculino & 51 & Casado & 3 & $\begin{array}{l}\text { Media } \\
\text { completa }\end{array}$ & FONASA & Más 5 años & Pudahuel \\
\hline 9 & Femenino & 46 & Casada & 3 & $\begin{array}{l}\text { Media } \\
\text { completa }\end{array}$ & FONASA C & Entre 1 y 5 años & La Reina \\
\hline 10 & Femenino & 62 & Soltera & 2 & $\begin{array}{c}\text { Media } \\
\text { completa }\end{array}$ & FONASA & Más 5 años & Santiago Centro \\
\hline 11 & Masculino & 78 & Casado & 2 & $\begin{array}{c}\text { Media } \\
\text { completa }\end{array}$ & FONASA & Entre 1 y 5 años & Providencia \\
\hline 12 & Masculino & 73 & Casado & 4 & $\begin{array}{l}\text { Media } \\
\text { completa }\end{array}$ & FONASA & Más 5 años & Lo Prado \\
\hline
\end{tabular}

Fuente: Elaboración propia.

ticipantes para validar las descripciones. Finalmente se revisó la literatura relevante al tema, para finalizar con la distribución de los resultados a la comunidad de enfermería. Se realizó triangulación de cada una de las resultados entre el equipo de investigación. El rigor metodológico se realizó de acuerdo a Noreña, utilizando los criterios de fiabilidad, validez, credibilidad, transferibilidad, consistencia y confirmabilidad ${ }^{8}$.

\section{Resultados}

Los participantes de este estudio lo conformaron 12 personas en terapia de sustitución renal de hemodiálisis (Tabla 1).

El significado de Calidad de vida para este grupo significó "seguir viviendo". En la experiencia están inmersos los familiares, el poder compartir el mayor tiempo y de la mejor manera posible junto a los seres queridos, en el cual la salud tiene relación con el bienestar físico y mental de los participantes, permitiendo realizar actividades de la vida diaria y ser personas autónomas.

"Yo por lo menos he dicho que esto es para siempre (...) vengo el lunes para poder vivir el martes, el día miércoles para poder vivir el jueves y el viernes para poder vivir el fin de semana...". (E3)

Seguir viviendo es la esencia de la experiencia dentro del relato de los participantes, los que refieren que producto de la terapia pueden continuar con su vida, lo aceptan de buena manera indicando que comprenden y están conscientes que necesitan realizar el tratamiento día por medio para poder vivir. Se destaca dentro de los aspectos positivos, que han mejorado su salud en relación a su estado previo a la hemodiálisis.

De esta manera el fenómeno de Calidad de Vida para los participantes se develó a través de tres categorías comprensivas: 1) Salud, 2) Familia y 3) Actividades de la vida diaria. 


\section{Salud}

El concepto de salud se presenta como un concepto amplio desde el punto de vista de los participantes, logrando identificar la influencia de la terapia de sustitución renal en ésta. De esta manera, la salud constituye la ausencia de la enfermedad o simplemente no estar en hemodiálisis. Sin embargo, algunos de los entrevistados describen cómo el tratamiento ha mejorado su estado de salud actual, a pesar de que la terapia tiene repercusiones físicas y emocionales.

"Bueno a mí, la verdad es que con la diálisis a mí me mejoró la calidad de vida porque antes esto era... cuando me di cuenta que estaba enfermo era muy mala, no podía ni hablar y... a mí me ha mejorado harto la calidad de vida" (E9).

La salud también es descrita desde la perspectiva de la salud mental, así, algunos de ellos manifiestan haber pasado por episodios depresivos y describen sentirse "amarrados a una máquina". Es difícil adaptarse a una nueva forma de vida y aceptar lo que la terapia restringe, se sufre en un principio pero con el tiempo logran asumir el proceso y lo toman con tranquilidad, intentado seguir una vida normal.

\begin{abstract}
"Me siento como amarrada, siempre he tenido esa sensación de amarrada, esa sensación de estar obligada, obligada a que hay que hacerlo no más poh" (E7).

"Que uno tiene que tomarlo normal, normal la diálisis para poder vivir, porque si uno se va en la depresión, no está bien, porque a mí me pasó también, a mí me dio la depre, tuve varios años con depresión, pero ahora yo superé la depre" (E8).
\end{abstract}

La salud, también está asociada a la relación que se establece con el equipo de salud, refiriendo un buen trato. Los entrevistados revelan que la atención de salud en este centro en particular es buena, tanto por parte de los médicos como del equipo de enfermería, aludiendo a la preocupación constante, dedicación y empatía que éstos demuestran hacia ellos.

"...a mí me gusta este centro, porque todas las enfermeras son como súper preocupadas, porque yo de repente como que me siento así con taquicardia y yo le digo y son como súper preocupadas..." (E1).

"Siempre me han atendido bien en todas partes, cuando a veces he ido de visita también, no, no, ningún problema, todas las niñas son amorosas" (E7).

\section{Familia}

Los entrevistados describen a la familia como el pilar fundamental a la hora de sobrellevar un tratamiento tan complejo y largo como la hemodiálisis. Desde la asistencia en la alimentación, la constante compañía y apoyo en las tareas de la vida diaria. Las personas revelan estar conscientes de la carga emocional que significan estas tareas, y del estrés que puede producir en sus cercanos el intentar adecuar sus vidas para ayudarlos, lo que en ocasiones podría mermar la relación familiar. Gran parte de los participantes indican llevar una vida familiar casi normal y manifiestan el deseo de permanecer junto a ellos el mayor tiempo posible.

"...en mi casa, me cuida mucho mi mujer, ella es la que me prepara ciertas comidas que me benefician y tengo mi hija que vive también conmigo que ella es la que me prepara todos los remedios..." (E3).

"Los familiares como que, de repente se sienten, se sienten como un poco, un poco presionados por tratar de estar contigo, de ayudarte y de repente ellos no, no, no lo pueden hacer, no tienen tiempo o... un montón de cosas, así que afecta, afecta harto" (E12).

\section{Actividades de la vida diaria}

Gran parte de las personas relatan dificultades físicas a la hora de realizar sus actividades cotidianas, aludiendo al cansancio la limitación de las tareas de la vida diaria, como cocinar, trasladarse de un lugar a otro, trabajar, etc. No obstante, algunos participantes señalan que la hemodiálisis ha logrado mejorar su estado de salud indicando que se sienten mejor y pueden realizar más cosas de las que hacían previo a la terapia.

"...estoy restringido, no salgo mucho, los mismos ejercicios que hacía antes ahora ya no, por ejemplo antes de mi casa me iba caminando hasta mi lugar de entretención, pero ahora ya tengo que ir en vehículo no más, no aguanto el training..." (E3).

"...a mí me permite hacer muchas cosas que no podía hacer sin la diálisis, así que yo encuentro que para mí por lo menos es muy positivo, y no quedo tan mal ni tan cansado, de hecho hago una vida casi normal, casi normal." (E9).

En cuanto a la alimentación, los entrevistados reconocen lo estricto que puede llegar a ser la dieta a la que 
deben someterse y la importancia que significa seguirla al pie de la letra, al igual que el tratamiento farmacológico, además indican las repercusiones de no respetarla y lo relevante de mantener un peso adecuado.

"...la alimentación es fundamental, tomarse todos los remedios que te dan, porque aquí te van controlando el peso, entonces se nota al tiro, pero eso sería, sobre todo la comida, porque uno según lo que coma es como se siente" (E3).

En relación al trabajo, las personas refieren que debido al cansancio y al tiempo que demanda la terapia ya no les es posible trabajar como antes y deben adaptar sus jornadas de trabajo o simplemente dejar de trabajar.

"En mi trabajo ha repercutido porque yo antes... trabajo en la feria, yo ya no puedo hacer lo que hacía antes. Por ejemplo, yo de repente hacía peso, tomaba cajas eso ahora no lo puedo hacer" (EIO)

"igual trabajo normal, pero cuando no..., vengo a la diálisis no trabajo, son otros días que trabajo, por ejemplo martes, jueves y sábado. Esos tres días trabajo" (El1).

Uno de los entrevistados describe como una repercusión de la terapia la abstinencia sexual, señalando que por esta causa, ya no tiene actividad sexual hace algunos años, evidenciando algunos mitos al respecto.

"Baja la parte de la sexualidad yo de los 14 años (en diálisis), llevo 3 años sin sexo, gracias a dios ella me comprende y ella por cuidar su riñón también no quiere sexo" (E5).

Otro de los participantes describe la importancia de la Fe y la creencia en Dios para poder sobrellevar este proceso y seguir adelante.

"Le pedí mucho al señor, al señor de los cielos, al señor Jehová, que yo quería vivir y entonces bueno y así me sumergí a las aguas, todo para volver a nacer y estar acá, entonces mediante Él y la diáli$\operatorname{sis}^{\prime \prime}(E 8)$.

\section{Resultados}

Mediante esta investigación se ha logrado comprender el significado de calidad de vida, mediante las propias vivencias que relataron los participantes entrevistados.
De acuerdo a la OMS, Calidad de vida constituye la percepción individual de lo que se vive en un contexto cultural específico, determinado por un sistema de valores en relación a lo que se espera de uno mismo ${ }^{4}$. Definición que dan cuenta los resultados del estudio, en tanto calidad de vida se ve influenciada por las vivencias de las personas, los objetivos que persiguen, el estado actual de la enfermedad, la autonomía y la relación con sus seres significativos. Esta investigación dio cuenta de tres áreas cruciales de la calidad de vida: la salud, familia y actividades de la vida, también descritas en la literatura, en cuanto a las dimensiones físicas, emocionales y sociales de la vida?.

Respecto de la salud, Zuñiga ${ }^{10}$ describe los aspectos físicos y mentales que afectan directamente la calidad de vida en concordancia con los resultados obtenidos en esta investigación. Los participantes refirieron la importancia de reponerse a la situación, ya que de lo contrario podrían sufrir cuadros de depresión, lo cual coincide con lo descrito en el estudio de $\mathrm{Cobo}^{11}$ et al., donde la mitad de los participantes describieron sentimientos de ansiedad y depresión. Aspectos que debieran pesquisarse de manera precoz en el marco de evaluaciones psico emocionales periódicas ${ }^{12}$. Por el contrario, otra investigación, señala las dimensiones de salud mental y rol emocional, como áreas con bajo impacto ${ }^{13}$. Por otro lado, los ingresos socioeconómicos y el nivel educacional, tendrían un efecto significativo en la calidad de vida de los participantes ${ }^{11}$, sin embargo, estos temas no fueron descritos por los participantes en este estudio.

La familia, constituye uno de los elementos centrales de la experiencia, reconociendo en ella un importante pilar para salir adelante y adaptarse a la terapia de hemodiálisis. Las mayores preocupaciones de las personas sometidas a hemodiálisis se centran en el apoyo y las relaciones familiares y la reincorporación laboral $^{14}$, lo que coincide con este estudio que demostró la importancia de la familia y el ámbito laboral en la calidad de vida de los entrevistados.

En relación a las actividades de la vida diaria, varios estudios han descrito las consecuencias en la calidad de vida, la dimensión del rol físico que sería una de los más afectados, seguida de la función social, aspectos descritos por los participantes como dificultades para desarrollar sus actividades domésticas e incorporarse en el ámbito laboral ${ }^{13,15}$. Otro aspecto de la vida diaria, lo configura las dificultades y cambios que deben realizar en cuanto a su alimentación ${ }^{14}$. Sadala ${ }^{16}$, describe que las personas asumen y reconocen las estrictas 
reglas que deben seguir bajo este tratamiento y que no hay otra solución posible si desean tener una vida más llevadera con su enfermedad. De esta manera, la importancia que tiene el personal de salud en la terapia, sería un eslabón clave para estas personas, puntos que convergen con esta investigación, reforzando el vínculo de esta relación usuario-equipo de salud. Por otra parte, de acuerdo a Borroto ${ }^{14}$ la sexualidad es uno de los aspectos importantes que se ven afectados en términos de la calidad de vida en personas en hemodiálisis, sin embargo, esta temática fue solamente abordada por uno de nuestros participantes.

Según Múñoz ${ }^{17}$, el valor que se da a la calidad de vida se ve influenciado por las comorbilidades y la edad, lo que se refleja en este estudio donde los pacientes con mayor edad y más enfermedades asociadas presentaron mayores problemas en ámbitos que requieren esfuerzo físico como el trabajo y las actividades de la vida diaria. Además, tal como lo indica Martínez ${ }^{18}$ en su estudio, existe una relación directa entre los días no saludables de los pacientes y los días sin salud física y sin salud mental que ellos presentan durante el mes, demostrando que la eficacia del tratamiento y la repercusión sobre la salud de los pacientes mejora el rendimiento físico y por ende su calidad de vida. Es importante además, comparar la calidad de vida de pacientes hemodializados y pacientes con trasplante renal, según Rodríguez ${ }^{19}$, el aspecto peor evaluado de un paciente trasplantado es el aspecto físico el cual está presente en la mayoría de quienes son sometidos a tratamiento con hemodiálisis. Así, como refiere Rebolledo ${ }^{20}$, el impacto de la terapia es altamente significativo, por lo que es relevante el tratamiento multidisciplinario para lograr el bienestar de los pacientes.

De esta manera, de acuerdo a los participantes el concepto de Calidad de vida lo significan como la posibilidad de seguir viviendo, destacando los aspectos positivos desde que se encuentran sometidos a hemodiálisis. Resultados similares son descritos Al-Arabi, en el cual describen la calidad de vida como una forma de estar vivo, gracias al amor de su familia y aceptar su condición como parte de su propia vida ${ }^{21}$.

En esta investigación se logró comprender lo que significa calidad de vida para pacientes que están sometidos a terapia de sustitución renal por hemodiálisis. Destacando como aspectos más relevantes para los participantes, su familia, la modificación de sus actividades de la vida, con el fin de poder prolongar la vida. Desde enfermería, se refuerza la necesidad de fortalecer la relación al momento de ejercer los cuidados y fomentar relaciones interpersonales armoniosas. Esta relación permite contribuir a un cuidado más seguro, de calidad e integral, considerando los aspectos que determinan la calidad de vida en este grupo, que de acuerdo a algunos autores se enfatiza en relaciones de largo plazo que permita este vínculo persona-enfermero ${ }^{21}$. El cuidar se configura como un acto ético, que involucra el respeto a la dignidad de la persona que requiere de dicho cuidado, la familia y la dignidad misma del enfermero que lo otorga ${ }^{22}$. Además implica la profesionalización del cuidado hacia la utilización de modelos y teorías de enfermería como herramientas éticas y de humanización de los cuidados.

A partir de estos resultados se sugiere continuar abordando el fenómeno de calidad de vida en usuarios en hemodiálisis en tanto se presenta como una problemática compleja, subjetiva y multidimensional. Es por ello que el abordaje debe ser integral, con el apoyo y acompañamiento de equipos multidisciplinarios que incluyan nutricionistas, psicólogos, asistentes sociales y acompañamiento espiritual, entre otros.

Las limitaciones de este estudio se refieren a la no transferibilidad de estos resultados a la población chilena, en tanto, más investigaciones son necesarias en esta área. Por otra parte sería de gran interés poder profundizar en Chile, aspectos relacionados a la sexualidad y la fe, temáticas poco abordadas por los participantes de este estudio. Así mismo, comprender las repercusiones en la calidad de vida en el contexto chileno y latinoamericano, podría contribuir a desarrollar mejoras en las estrategias de cuidado desde un enfoque más amplio, biopsicosocial y holístico.

\section{Agradecimientos}

Agradecer al Centro de Diálisis Nuñoa y su personal por su hospitalidad y colaboración en el estudio, en especial a Isabel Álvarez, enfermera coordinadora, por toda su ayuda y disposición.

Recibido: 15 enero 2016

Revisado: 29 enero 2016

Modificado: 28 febrero 2016

Aceptado: 3 marzo 2016 


\section{Bibliografía}

1. National Kidney Foundation. EEUU. (Acceso 15 Diciembre 2015). Disponible: https://www.kidney. org/kidneydisease/aboutckd\#ckd

2. Ministerio de Sanidad Servicios Sociales e Igualdad. Documento Marco sobre Enfermedad Renal Crónica (ERC) dentro de la Estrategia de Abordaje a la Cronicidad en el SNS. España. 2015. (Acceso 15 Diciembre 2015) Disponible: http://www.msssi.gob.es/organizacion/sns/ planCalidadSNS/pdf/Enfermedad_Renal_ Cronica_2015.pdf

3. Poblete H. XXXIV Cuenta de Hemodiálisis Crónica en Chile. (Acceso 20 Septiembre 2015). Disponible: http://fmc-ag.cl/_file/file_54_ cuenta\%20hemodialisis\%202015.pdf

4. Urzúa, A. Caqueo-Urízar, A. Calidad de vida: Una revisión teórica del concepto. Sociedad Chilena de Psicología Clínica. Ter Psicol. 2012; 30 (1): 61-71.

5. Nunes C, Lobo A. Estudio de las dimensiones de calidad de vida en pacientes hemodializados. Rev Enferm. 2012; 3(8): 39-45.

6. Seguí $A$, Amador P, Ramos AB. Calidad de vida en pacientes con insuficiencia renal crónica en tratamiento con diálisis. Rev Soc Esp Enferm Nefrol. 2010; 13(3): 1139-1375.

7. Streubert H, Carpenter D. Qualitative research in Nursing: Advancing the humanistic imperative. Philadelphia: Editorial Lippincott Williams \& Wilkins; 2011.

8. Noreña A, Alcaraz-Moreno N, Rojas JG, ReboIledo-Malpica D. Aplicabilidad de los criterios de rigor y éticos en la investigación cualitativa. Rev Aquichan. 2012; 12(3): 263-274.

9. Fernández-López JA, Fernández-Fidalgo $M$, Cieza A. Los conceptos de calidad de vida, salud y bienestar analizados desde la perspectiva de la Clasificación Internacional del Funcionamiento (CIF) Rev Esp Salud Pública 2010; 84: 169-184.

10. Zúñiga C. et al. Evaluación de la calidad de vida en pacientes en hemodiálisis crónica mediante el cuestionario "Kidney Disease Quality of Life (KDQOL-36)". Rev Med Chile. 2009; 137(2): 200-207.

11. Cobo. Factores sociológicos y calidad de vida relacionada con la salud en pacientes en hemodiálisis. Rev Soc Esp Enferm Nefrol 2011; 14 (2): 98-104.

12. García H, Calvanese N. Calidad de vida percibida, depresión y ansiedad en pacientes con tratamiento sustitutivo de la función renal. Psicología y Salud 2008; 18 (1): 5-15.

13. Albañil T, Ramírez M, Crespo R. Análisis de la calidad de vida en pacientes en hemodiálisis ambulatoria y su relación con el nivel de dependencia. Rev Enferm Nefrol. 2014; 17 (3). (Acceso 15 Diciembre 2015). Disponible en: http://scielo.isciii.es/ scielo.php? pid=S225428842014000300002\&script=sci_arttext

14. Borroto G. et al. Percepción de la calidad de vida por enfermos sometidos a tratamientos de hemodiálisis o trasplante renal. Estudio comparativo. Rev Cubana Med. 2007; 46 (3). (Acceso 15 Diciembre 2015). Disponible en: http://bvs.sld.cu/revistas/med/vol46_3_07/ med04307.html

15. Oliveira M, Arruda D. Percepción de las personas con insuficiencia renal crónica sobre la calidad de vida. Enferm glob. 2012; 11(28). (Acceso 20 Diciembre 2015). Disponible en: http://scielo.isciii.es/scielo.php?pid=S169561412012000400014\&script=sci_arttext

16. Sadala $M$, Lorençon $M$. Vivir con una máquina de hemodiálisis. EDTNA/ERCA Journal of Renal Care (Spanish). 2006; 32(3): 152-157.

17. Muñoz $R$, Oto $A$, Barrio $R$, Fernández $M$. Evolución de la calidad de vida en pacientes en hemodiálisis: estudio prospectivo a un año. Servicio de Nefrología, Hospital Universitario Miguel Servet, Zaragoza 2006; 9(1). (Acceso 20 Diciembre 2015). Disponible en: $\quad$ http://www.revistaseden.org/imprimir. aspx?idArticulo=4424170098093097424170

18. Martínez C,ValenciaA.Calidad de vida del paciente nefrópata en programa de hemodiafiltración. Rev 
Mex de Enfermería Cardiológica, 2010; 18(1-2): 7-12.

19. Rodríguez M. et al. Calidad de vida y trasplante renal en mayores de 65 años. Rev Soc Esp Enfer Nefrol, 2009; 12(1): 26-30.

20. Rebollo A, Morales J, Pons M, Mansilla J. Revisión de estudios sobre calidad de vida relacionada con la salud en la enfermedad renal crónica avanzada en España. Rev Esp Nefrología, 2015; 35(1): $92-$ 109.
21. Al-Arabi S. Quality of Life: Subjective Descriptions of Challenges to Patients with End Stage Renal Disease. Nephro Nursing Journal, 2006; 33 (3): 285-293.

22. Avilés L, Soto C. Modelos de Enfermería en Unidades de Paciente Crítico: un paso hacia el cuidado avanzado. Enferm glob.2014; 34(4):323329. 